Universidad de Talca - Facultad de Ciencias Jurídicas y Sociales Teoría del derecho, Alejandro Vergara Blanco. Thomson Reuters; Santiago de Chile, 2018, 245 pp. Antonio Pedrals

\title{
Teoría del derecho, Alejandro Vergara Blanco. Thomson Reuters; Santiago de Chile, 2018, 245 pp.
}

Antonio Pedrals*

1) En un ambiente como el jurídico, de progresiva y creciente especialización, se recibe con alegría el texto "Teoría del derecho. Reglas y principios, jurisprudencia y doctrina", que entrega el profesor Alejandro Vergara Blanco, titular de Teoría del Derecho y también de Derecho Administrativo en la Facultad de Derecho de la Pontificia Universidad Católica de Chile.

Sin perjuicio de su interés general, el libro se ha preparado como material de apoyo para la cátedra de Teoría del Derecho, que se imparte en el primer año de la carrera en la universidad mencionada, subrayándose que no se trata de una mera "introducción al derecho", sino del desarrollo de una disciplina específica que, por otra parte, no debe confundirse con filosofía del derecho'.

Tras una introducción sobre el método de la enseñanza jurídica, la obra comprende siete partes: 1 . Una teoría del derecho para la era democrática y neomoderna; 2. Distingos entre teoría, filosofía y doctrina jurídicas; 3 . Normas o reglas; 4. Principios jurídicos; 5. Jurisprudencia; 6 . Doctrina, y 7 . La summa divisio de lo público y privado.

Reuniendo 245 páginas en total, el texto entrega además bibliografía, nóminas de normas y nombres citados, y un índice detallado que complementa al inicial.

2) Como se ha dicho, el autor discurre sobre la base de que la teoría del derecho (que otros llaman teoría general del derecho, ciencia general del derecho, doctrina general del derecho, etc.) posee una suerte de autonomía que permite diferenciarla de la filosofía del derecho y de la asignatura de introducción; todo ello sin perjuicio de las necesarias interrelaciones que deben reconocerse entre las disciplinas.

\footnotetext{
* Profesor de la Universidad de Valparaíso. Teoría General del Derecho. Doctor en Derecho por la Universidad Complutense de Madrid. Correos electrónicos: antonio.pedrals@uv.cl; pedrals.antonio@ gmail.com.

${ }^{1}$ Vergara (2018), pp. 5, 6, 41, 44 y 45.
} 
Al respecto - dice el autor- cabe evitar dos usuales confusiones: “a) confusión con el sucedáneo curso chilensis de 'Introducción al Derecho' y b) confusión con la filosofía del derecho, cuyo núcleo disciplinario es distinto al derecho (partiendo por el dato epistemológico de que esa disciplina es parte de la "filosofía", y no del "derecho"). Ello, no obstante la alta relevancia que cabe reconocer a la filosofía del derecho en la formación jurídica en los valores; igualmente es el caso de otras disciplinas fronterizas, como la historia del derecho o la sociología del derecho, muy relevantes para la enseñanza jurídica" ${ }^{2}$.

La filosofía del derecho en sus distintas tradiciones, muchas en disputa, ofrecería entonces "una mirada externa del fenómeno jurídico, y es el jurista y el teórico el que, mediante un tamiz metodológico bien refinado, determina la magnitud y utilidad del uso de categorías filosóficas al Derecho; pero no al revés: no es el filósofo el que, en un intento de colonización de un territorio que le es ajeno, podría dominar con sus métodos y doctrinas extraños en una especie de manipulación de las fuentes del Derecho" ${ }^{\prime 3}$.

El criterio señalado coincide con el de otros autores que aceptan este predicamento, aun sabiendo que las discusiones sobre el punto no son escasas ${ }^{4}$. En efecto, hay analistas que consideran que: a) La teoría del derecho (en adelante TD) es la filosofía del derecho ${ }^{5}$. b). La TD es una rama de la filosofía del derecho $^{6}$. c). La TD es una rama mixta, filosófico-científica ${ }^{7}$. d). Procede distinguir diversas TD: una filosófica y otra científica ${ }^{8}$, etc.

En concordancia con el planteamiento que expone, el autor sostiene que una buena formación jurídica no debe prescindir de estos estudios, reconociendo que, en Chile, este no es todavía un criterio completamente aceptado ${ }^{9}$; todo

2 Vergara (2018), pp. 1 y 2.

${ }^{3}$ VerGara (2018), pp. 44 y 45.

4 Barcellona y Cotturri (1976), p. 31; Bobbio (1980), pp. 71 y ss., 80 y ss.; Consentini (1930), p. 26; díaz (1974), p. 73; García Belaunde (1982), pp. 71 y ss.; García Maynez (1974), pp. 11 y ss.; Guasp (1957), pp. 145 y ss.; Haesaert (1948), p. 27; Jiménez de Asúa (1976), p. 27; LeCLerCQ (1966), pp. 83 y ss.; NaWiasky (1962), p. 26; Piovani, Linee di una filosofia del diritto, citado por García MaYnez (1974), p. 15; Reyes (1983), p. 127; RodríGuez-Arias (1961), pp. 61 y ss.; Squella (1977), p. XIX; Viera (1964), pp. $5,21,28$ y ss.

5 Ross (1970), p. 1. Ver también la posición de Legaz, citada en Serrano (1966), p. 41, y De Rivacoba (1982), p. 191.

${ }_{6}^{6}$ Bobbio (1980), pp. 71 y ss.; Recasens (1965), p. 160; De Rivacoba (1982), p. 191.

7 Carnelutti, citado por Bobbio (1980), p. 74.

${ }^{8}$ Boвbio (1980), p. 74.

${ }^{9}$ Vergara (2018), p. 49. 
ello a pesar de que continuamente está apareciendo bibliografía pertinente, con textos recientes o reeditados ${ }^{10}$.

En lo que toca al aspecto pedagógico, nos parece relevante tener en cuenta la experiencia iniciada en la Universidad de Chile en la década del setenta del siglo pasado, y prolongada luego, hasta nuestros días, en la Universidad de Valparaíso, continuadora de la antigua sede de la Universidad de Chile en dicha ciudad. A ello nos referiremos más adelante.

3) A la inversa de quienes estudian al derecho como una realidad en sí, aislado de su "circunstancia", a veces de modo intemporal, el autor considera el derecho de la época actual, al que califica de democrático y "neomoderno". Señala que, desde el siglo XIX hasta hoy, el derecho democrático sería el característico de casi todo Occidente y que, a la vez, este derecho tendría un carácter "neomoderno".

En el caso de la cultura nacional, "Ios postulados fundadores del Derecho moderno han sido objeto de una metamorfosis que ha estado puesta ante nuestros ojos en los últimos casi 40 años con mucha nitidez: las fuentes del Derecho han mutado, y ya no todo es ley; la realidad jurídica (que es base de cada disciplina del Derecho, en donde se anidan las relaciones jurídicas) ha mutado, y ya no todo depende de la voluntad de algún órgano de la administración del Estado. Respectivamente, otra fuente y otro fenómeno social, alteran los monopolios de la ley y de la administración del Estado: los principios se ponen ahora al lado y dominan junto a la ley; el mercado se pone también ahora al lado y domina junto a la administración del Estado"11.

4) La obra se funda en que el derecho sería, ontológicamente, una realidad constituida por cuatro dimensiones:

"Da la impresión que al analizar el Derecho nos estamos refiriendo exclusivamente a las reglas jurídicas contenidas en las normas; sin embargo, ello no es así: en la empiría del Derecho contemporáneo, existe un escenario cuádruple de fuentes del Derecho vigentes, el que resulta de sus sendas sedes de producción, todas culturales y temporales, fruto de desarrollos o acuerdos actuales". En definitiva, el derecho se reduce a cuatro fenómenos: las leyes, el hecho jurídico, la jurisprudencia y la doctrina ${ }^{12}$.

La observación de estas cuatro dimensiones no solo permite trazar el campo epistemológico del derecho y de la teoría del derecho, sino que tiene una

\footnotetext{
10 Aguiló (2012), p. 256; Amunátegui (2016), p. 140; Bobbio (2017), p. 284; Correa (2016), p. 323. Valderrama et al. (2013), p. 490; Misari Torpoco (2013), p. 237; Millard (2016), p. 180; Nino (2014), p. 200; Peña (2011), p. 170; Moderne (2017), p. 296; Rojas y Moreno (2015), p. 272.

11 VerGara (2018), p. 12.

12 Vergara (2018), p. 11. Cursiva nuestra.
} 
consecuencia pedagógica: "saca a la luz los seis temas y teorizaciones esenciales de toda Teoría del Derecho (los cuales no puede soslayar ningún curso ni texto general sobre esta materia): i. normas o reglas; ii. costumbres; iii. jurisprudencia; iv. doctrina; v. interpretación de las leyes; y, vi. principios. Todos los cuales, en el orden que corresponde tienen cabida en este libro"13.

El autor toma aquí posición frente al debatido problema de la consistencia del derecho, tema no sencillo, sobre el que, como se sabe, existen múltiples enfoques. Recordemos, por ejemplo, las doctrinas normativistas ${ }^{14}$, conductistas $^{15}$, dimensionalistas ${ }^{16}$, institucionalistas ${ }^{17}$, etc.

5) Sin perjuicio de reconocer que, tal como señala el autor, las fuentes jurídicas integran el derecho (o, si se prefiere, la realidad jurídica), conviene tener presente que diversos argumentos doctrinarios postulan que el derecho no se agota en las fuentes ${ }^{18}$.

Basta un análisis superficial del fenómeno jurídico, dice Goldschmidt, para descubrir elementos de diferente índole. "Si pasamos revista a lo que suele Ilamarse 'lo jurídico', nos encontramos, en primer lugar, con una congerie de conductas llevadas a cabo por jueces, secretarios, funcionarios, abogados; en segundo lugar, nos conmueven estas conductas, sea que su justicia nos complazca y reconforte, sea que su injusticia nos indigne y subleve; en tercer lugar, nos enteramos de lo jurídico cuando estudiamos los códigos del país y el sin fin de manuales y tratados dedicados a su análisis"19.

Por su lado, Ortega, con fundamento e incluso con humor, señala que "Io jurídico" del derecho es solo una parte de su efectiva realidad, cuya otra parte son una porción de cosas de la vida de un pueblo que no parecen tener nada que ver con él ${ }^{20}$. Según el maestro español, "el Derecho se compone de más cosas que una idea: por ejemplo, forman parte de él los bíceps de los gendarmes o

\footnotetext{
13 Vergara (2018), p. 13.

14 Para algunos intérpretes, un ejemplo de concepción normativista se encontraría en la conocida (y trabajada) definición que da Couture de derecho. COUTuRE (1960), p. 229.

15 Aquí puede mencionarse la escuela egológica del derecho, de Carlos Cossio y sus discípulos.

${ }^{16}$ Doctrinas de Miguel Reale, Miguel Villorro, Werner Goldschmidt, etc.

17 Doctrinas de Santi Romano, Maurice Hauriou, Joaquín Ruiz Jiménez, George Renard, etc.

18 Pedrals (2014), pp. 24 y ss.

19 Goldschmidt (1962), p. 27, cursiva nuestra. La palabra "congerie", que emplea el autor, significa acumulación, amontonamiento. Véase también el texto de ECHEVERRía (1986), p. 378, sobre la teoría "impura" del derecho.

20 Ortega (1962), p. 159; Garrigues (1971), p. 99; Truyol (1998), p. 14.
} 
sucedáneos. A la técnica del puro pensamiento jurídico tienen que acompañar muchas otras técnicas aún más complicadas"21.

Agreguemos que estas y otras posiciones análogas se recogen, de algún modo, integradamente, en esa "nueva visión" que se ha ido desarrollando para dirigir la marcha de la ciencia en general y a la que se alude como perspectiva sistemática, holística, compleja, multidimensional, etc., tema que aquí solo cabe mencionar ${ }^{22}$.

6) Sin perjuicio de concordar con lo que expone el autor acerca de temas de la teoría del derecho ( $\left.N^{\circ} 4\right)$, consideramos que el campo de esta disciplina, según lo señalado en el número 5 que precede, podría ampliarse teniendo en cuenta, por ejemplo, entre otras, las siguientes materias:

- Los grandes objetivos civilizacionales ${ }^{23}$. Variados estudiosos de las posibles salidas del "laberinto" sociohistórico de la actualidad se plantean, entre otros aspectos, la idea nada sencilla de hacer un inventario de los problemas más relevantes, para luego intentar establecer objetivos que sirvan para orientar a Estados, organizaciones e individuos. El punto ha sido afrontado por diversas manifestaciones culturales y por el propio derecho, según consta en declaraciones y tratados de los últimos cincuenta años y en diversas disposiciones jurídicas. Para el profesor Oscar Sarlo, de la Facultad de Derecho de la Universidad de la República, Uruguay, estos "grandes objetivos civilizacionales" constituyen el problema central de los estudios jurídicos generales de la actualidad ${ }^{24}$.

- Los procesos jurídicos generales ${ }^{25}$. De acuerdo a una perspectiva amplia, puede estimarse que el derecho está constituido por diversos procesos relativos a la existencia de las fuentes, a la proyección social de las mismas, al apoyo de dicha protección social y al análisis crítico de procesos y fuentes. Además, junto a los procesos jurídicos "regulares", existen también "procesos extrains-

\footnotetext{
21 Ortega (1955), p. 294. Siempre dentro de una concepción amplia del derecho, cabe citar, entre otras, la posición del exmagistrado del Tribunal Supremo español Carlos de la Vega Benayas, que, a través de su experiencia doctrinaria, docente y judicial resume su pensamiento en el dístico "el hombre, más la norma, más el hecho/ eso es lo que entiendo por derecho". De LA VEGA (1976), p. 116.

22 Pedrals (2014), pp. 24 y ss.; Gómez (2018), p. 134. En este último trabajo, que debemos a la destacada académica Taeli Gómez Francisco, especializada en estos temas, leemos lo siguiente: "En definitiva, el paradigma científico de la complejidad contribuye a nuevas reflexiones para las ciencias dedicadas a la educación, como la pedagogía. También favorece relaciones conscientes y dialogantes entre esta y las disciplinas de la didáctica y psicología, para revisar, epistemológicamente, la coherencia que debe existir entre objetos de estudios cada vez más complejos, sistémicos e inciertos, y así advertir las debilidades de paradigmas mecanicistas, lineales, atomistas, fraccionadores y simplificadores".

23 Pedrals (2014), pp. 43 y ss.

${ }^{24}$ Carta al autor de este trabajo.

25 Pedrals (2014), pp. 139 y ss.
} 
titucionales", es decir, actuaciones informales que interfieren en los procesos regulares y que, en consecuencia, tienden a alterar la "normalidad" jurídica.

- Los factores que intervienen en los procesos son de distinto carácter y pueden clasificarse en individuales, sociales, culturales, técnicos, materiales, etc., sin olvidar al azar; Cicerón solía decir que es el azar, y no la prudencia, el que rige en verdad la existencia ${ }^{26}$.

- El análisis crítico del derecho ${ }^{27}$. Llamamos análisis crítico, en general, al proceso que consiste en contrastar una determinada realidad con un criterio que, en algún sentido, implica un deber ser. Al respecto son ampliamente conocidos los criterios de juridicidad, socialidad, justicia, racionalidad y seguridad. Algunos autores agregan un "criterio estético", aunque sea un tema que suele menospreciarse en nuestro ámbito, "como si se tratara de algo secundario" 28 .

7) Por otra parte, resulta de interés destacar que el autor no es, como tantas veces ocurre con los generalistas, un jurista que observa el derecho "desde fuera", sino un profesional que ejerce prácticamente su carrera, cultivando estudios jurídicos generales junto a disciplinas especializadas. Estimamos que esta perspectiva le confiere a su trabajo un especial valor teórico-práctico y le evita seguir

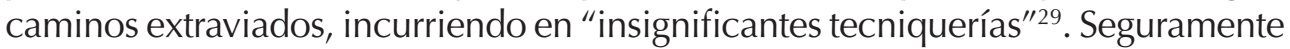
la cátedra de Teoría del Derecho le ha sido personalmente útil para el ejercicio de la abogacía, así como dicho ejercicio le ha sido beneficioso para la cátedra, al trabajar con una realidad de "carne y hueso", no meramente libresca.

Otro punto que procede subrayar es la versación humanística del autor, que se revela en variadas referencias literarias, filosóficas, etc., que constituyen un aporte adicional para la comprensión de la compleja y tantas veces enigmática realidad que, para simplificar, Ilamamos derecho ${ }^{30}$.

8) Volvemos, finalmente, a un punto aludido más arriba ( $\left.N^{\circ} 2\right)$ que, en este contexto, nos parece de interés. En la década del setenta del siglo pasado, la Facultad de Derecho de la Universidad de Chile incorporó, prescriptivamente, en el último nivel de la carrera (paralela a Filosofía del Derecho) una asignatura de Teoría General del Derecho, que debería asumir, en la medida de lo posible, una visión científica, general y multidimensional del fenómeno jurídico.

\footnotetext{
26 Pedrals (2014), pp. 165 y ss.

27 Pedrals (2014), pp. 377 y ss.

28 Llewellyn (1962), p. 19.

29 Ramos Sobrino (1969), p. 38.

30 Encontramos, por ejemplo, referencias a Borges, Shakespeare, Prado, Kant, etc. Ver VerGARA (2018), pp. $10,13,27,38,52$ y 149 .
} 
En Santiago, los primeros profesores de la asignatura fueron dos conocidos académicos: don Aníbal Bascuñán Valdés y don Eduardo Novoa Monreal, que impartieron cursos con diversas inspiraciones; el primero con acento en la ciencia jurídica, y el segundo apuntando fundamentalmente a problemas sociales vinculados con el derecho. En la Escuela de Valparaíso el profesor a cargo fue el autor de estas líneas.

Por diversas circunstancias que no son el caso, la cátedra subsistió solo en la Escuela de Valparaíso, donde se ha mantenido durante más de cuatro décadas, generando publicaciones doctrinarias ${ }^{31}$, memorias de prueba ${ }^{32}$, jornadas de estudio nacionales ${ }^{33}$, workshops en el extranjero ${ }^{34}$ y un congreso multidisciplinario nacional sobre "El derecho en el siglo XX"35.

Un panorama actual del contenido de la asignatura puede verse en una obra que, precedida de diversos trabajos parciales, se dio a la imprenta en el año $2014^{36}$.

\section{BibliOgRAFÍA CITADA}

Aguiló Regla, Josep (2012): Teoría general de las fuentes del derecho (Barcelona, Ariel).

Amunátegui Perello, Carlos (2016): Teoría y fuentes del derecho (Santiago de Chile, Ediciones UC).

\footnotetext{
31 Pedrals (2014), pp. 545 y ss.

32 Más de sesenta trabajos.

33 La Primera Jornada de Ciencia General del Derecho se llevó a cabo en 1979 y versó sobre El Precedente. La segunda (1980) trató sobre La equidad en el derecho chileno. La tercera (1981), sobre Los principios generales del derecho en el ordenamiento chileno. La cuarta (1982), sobre El principio de legalidad en el ordenamiento positivo. La quinta (1983), sobre Desajuste entre norma y realidad. La sexta (1984), sobre Ardides, argucias y astucias en el campo del derecho. La séptima (1985), sobre Actos lícitos e ilícitos. La octava (1986), sobre Los incentivos en el campo del derecho. La novena (1987), sobre Problemas que ofrece el sentido del texto. La décima (1988), sobre "¿Nuevos conceptos e instituciones jurídicas?". La decimoprimera (1989), sobre Pensando el nuevo derecho. La decimosegunda (1990), sobre Las nuevas técnicas biomédicas ante los estudios generales del derecho. La decimotercera (1992), sobre El mundo actual. La decimocuarta (1993), sobre La cooperación en el campo jurídico.

${ }^{34}$ Ellos se propusieron y realizaron en el Instituto Internacional de Sociología Jurídica de Oñati, País Vasco, España, y trataron sobre los siguientes temas: Nueva conciencia del derecho (1996), Nuevos modelos de cooperación social (2002), Nuevos modelos de vida personal y comunitaria (2004) e Iniciativas individuales para el mejoramiento de la convivencia (2006).

${ }^{35}$ El congreso se celebró en 1991 para conmemorar los ochenta años de la Escuela de Derecho de la Universidad de Valparaíso.

36 Pedrals (2014), p. 589.
} 
Barcellona, Pietro, y Coturrl, Giuseppe (1976): El Estado y los juristas (Traducc. Juan Ramón Capella, Barcelona, Editorial Fontanella).

Bоввı, Norberto (1980): Contribución a la teoría del derecho (Traducc. Alfonso Ruiz Miguel, Valencia, Fernando Torres Editor).

(2017): Teoría general del derecho (Traducc. Eduardo Rozo Acuña, Bogotá D.C., Editorial Temis).

Cosentinı, Francesco (1930): Filosofía del derecho (México D.F., Editorial Cultura).

Correa V., Rodolfo (2016): Teoría general del derecho (Medellín, Biblioteca Jurídica Diké).

Couture Etcheverry, Eduardo J. (1960): Vocabulario jurídico (Montevideo, Biblioteca de Publicaciones de la Facultad de Derecho y Ciencias Sociales de la Universidad de la República).

De La Vega Benayas, Carlos (1976): Teoría, aplicación y eficiencia en las normas del Código Civil (Madrid, Editorial Civitas).

De Rivacoba y Rivacoba, Manuel (1982): "Proyecciones de la teoría pura en el pensamiento penal", en: AA. VV., Apreciación crítica de la teoría pura del derecho (Valparaíso, Edeval), pp. 181-192.

Díaz García, Elías (1974): Sociología y filosofía del derecho (Madrid, Taurus).

ECHEVERRía YÁÑez, José (1986): "Lecciones preliminares de teoría general del derecho y del cambio social", en: Revista Jurídica de la Universidad de Puerto Rico (Vol. LV, No 3), pp. 371-446.

García Belaunde, Domingo (1982): Conocimiento y derecho (Lima, Universidad Católica del Perú).

García Maynez, Eduardo (1974): Filosofía del derecho (México D.F., Porrúa).

Garriguez Díaz-Cañabate, Joaquín (1971): Hacia un nuevo derecho mercantil (escritos, lecciones y conferencias) (Madrid, Editorial Tecnos).

GoldSCHMIDT, Werner (1962): Introducción al derecho (estructura del mundo jurídico) (Buenos Aires, Aguilar Editor).

Gómez Francisco, Taeli (2018): La complejidad: Un paradigma para la educación (Copiapó, Universidad de Atacama Editores).

Guasp Delgado, Jaime (1957): "Exactitud y derecho", en: Anuario de Filosofía del Derecho $\left(\mathrm{N}^{\circ} \mathrm{V}\right)$, pp. 109-149.

HAESAERT, Jean (1948): Theorie generale du Droit (Bruselas, Emile Bruylant). Jiménez de Asúa, Luis (1976): La ley y el delito (Buenos Aires, Sudamericana).

LECLERCQ, Jacques (1966): Introducción a las ciencias sociales (Traducc. JoséÁngel de Juanes, Madrid, Guadarrama). 
LLEWELLYN, Karl (1962): Jurisprudence. Realism in Theory and Practice (Chicago, The University Of Chicago Press).

Millard, Eric (2016): Teoría general del derecho (Bogotá D.C., Universidad del Externado de Colombia).

Misari Torpoco, David (2013): Teoría general del derecho (Perú, Asociación Peruana de Ciencias Jurídicas y Conciliación).

Moderne, Franck (2017): Principios generales del derecho. Método y aceptación en derecho administrativo y constitucional (Traducc. Alejandro Vergara Blanco, Santiago de Chile, Thomson Reuters).

NAWIASKY, Hans (1962): Teoría general del derecho (Traducc. José Zafra Valverde, Madrid, Rialp).

Nino, Carlos Santiago (2014): Derecho, moral y política: Una revisión de la teoría general del derecho (Buenos Aires, Siglo XXI Editores).

Ortega y Gasset, José (1955): Obras completas de José Ortega y Gasset (Madrid, Revista Occidente), Tomo IV (1929-1933).

(1962): Obras completas de José Ortega y Gasset (Madrid, Revista Occidente), Tomo XI (1960-1962).

Pedrals de Cortázar, Antonio (2014): Fundamentos de teoría general del derecho (Santiago de Chile, Librotecnia).

Peña Peña, Rogelio (2011): Teoría general del derecho (Bogotá D.C., Ecoe Ediciones).

Ramos Sobrino, Blas (1969): El derecho y el hombre actual (Madrid, Gráficas Halar).

ReCASEns Siches, Luis (1965): Tratado general de filosofía del derecho (México D.F., Porrúa).

Reyes Soto, Nelson (1983): "Respuesta a la encuesta sobre filosofía del derecho", en: Anuario de Filosofía Jurídica y Social (No 1), pp. 126-130.

Rodríguez-Arias Bustamante, Lino (1961): Ciencia y filosofía del derecho (Buenos Aires, Ediciones Jurídicas Europa-América E.J.E.A.).

Rojas, Jorge, y MOReno, Romina (2015): Derecho procesal y teoría general del derecho (Santa Fe, Rubinzal Culzoni).

Ross, Alf (1970): Sobre el derecho y la justicia (Traducc. Genero R. Carrió, Buenos Aires, Editorial Eudeba).

Serrano Villafañe, Emilio (1966): La filosofía del derecho y la teoría general del derecho en la actualidad (Madrid, Reus). 
Squella Narduccl, Agustín (1977): Derecho, desobediencia y justicia (Valparaíso, Edeval).

Truyol y SerRA, Antonio (1998): "Salvador Lissarrague Novoa (1910-1967)", en: Lissarrague, Salvador, Bosquejo de teoría social (Madrid, Editorial Tecnos), pp. 9-38.

ValderRama Bedoya, Francisco et al. (2013): Teoría del derecho (Medellín, Sello Editorial Universidad de Medellín).

Vergara Blanco, Alejandro (2018): Teoría del derecho. Reglas y principios, jurisprudencia y doctrina (Santiago de Chile, Thomson Reuters).

Viera RUIZ, Luis (1964): "Realismo e idealismo en la teoría general del derecho", en: Revista El Derecho ( $N^{\circ}$ 89), pp. 5-32. 